\title{
Mode transformer for hard-surface waveguides
}

\author{
T. Uusitupa, A. Viitanen \\ Electromagnetics Laboratory \\ Department of Electrical and Communications Engineering \\ Helsinki University of Technology, P.O. Box 3000, FIN-02015 HUT, Finland \\ e-mail: tero.uusitupa@hut.fi, ari.viitanen@hut.fi
}

\begin{abstract}
Mode transformation effect inside a corrugated circular waveguide filled with gyrotropic material, e.g., magnetoplasma or ferrite, is considered. The corrugation is in axial direction and the depth of the corrugation is a quarter wave length forming a boundary condition equal to a hard surface (HS). It is assumed that the material is slightly anisotropic and gyrotropic. This can be achieved at certain frequency range with a proper axial magnetic field strength. The eigenwaves are elliptically polarised hybrid-mode fields, which propagate with slightly different propagation factors. This difference in propagation factors causes the mode transformation.
\end{abstract}

\section{INTRODUCTION}

The tuned corrugated waveguides are used in microwave applications where special kind of properties for field propagation are needed, for example, in antenna horn feed [1]. In corrugated waveguide there can propagate $T E, T M$ and elliptically polarised fields, and in hard-surface waveguide also TEM fields [2]. In this study the corrugation is in the axial direction, thus, forming the boundary condition for hard-surface waveguide. Instead of corrugation, an alternative way to implement hard-surface is to use dielectric layer loaded with thin conducting strips [3]. Time harmonic fields (depending on $t$ as $e^{j \omega t}$ ) are considered and the propagating fields depend on $z$ as $e^{-j \beta z}$, where the propagation factor $\beta$ is a real number. A mode transformer for a HS waveguide has previously been studied in [4], where chiral medium has been used. In this paper practically more interesting application example is studied. The HS waveguide is filled with ferrite material biased with static magnetic field which is in $z$-direction. Only a small gyrotropy is reguired. Schematic Figure 1 shows HS waveguide with some notations used.

\section{THEORY}

The constitutive relations and material parameters for ferrite are

$$
\begin{aligned}
\mathbf{D} & =\epsilon \mathbf{E}, \quad \mathbf{B}=\left[\mu_{t} \overline{\bar{I}}_{t}+\mu_{z} \mathbf{u}_{z} \mathbf{u}_{z}-j \mu_{g} \mathbf{u}_{z} \times \overline{\bar{I}}\right] \cdot \mathbf{H} . \\
\mu_{t} & =\mu_{o}\left(1+\frac{\omega_{o} \omega_{m}}{\omega_{o}^{2}-\omega^{2}}\right), \quad \mu_{z}=\mu_{o}, \quad \mu_{g}=\mu_{o} \frac{\omega \omega_{m}}{\omega_{o}^{2}-\omega^{2}}
\end{aligned}
$$

where $\omega_{o}=\gamma B_{o}$ is the Larmor precession frequency ( $B_{o}$ is the strength of the static magnetic flux density in $z$-direction), $\omega_{m}=\mu_{o} \gamma M_{s}, \gamma$ is the gyromagnetic ratio and $M_{s}$ is the saturation magnetization [5]. For magnetoplasma constitutive relations are of similar form i.e. one can use dyadic permittivity model. In terms of theoretical analysis, these two media, ferrite and magnetoplasma, are quite similar and can be obtained by duality from each other. In this study the focus is on ferrite material.

First step of the analysis is to find the propagating eigenfields depending on $z$ as $e^{-j \beta z}$ and the corresponding propagation factors. The electric and magnetic fields are written with transverse and axial parts as $\mathbf{E}=\mathbf{e}+E_{z} \mathbf{u}_{z}$ and $\mathbf{H}=\mathbf{h}+H_{z} \mathbf{u}_{z}$ and are inserted into the Maxwell equations $\nabla \times \mathbf{E}=-j \omega \mathbf{B}$ and $\nabla \times \mathbf{H}=j \omega \mathbf{D}$. Using the small gyrotropy assumption i.e., $\mu_{g} / \mu_{t}$ small, and also assuming that $\beta$ is not very near $k_{t}, \beta<k_{t}$, elimination of transverse fields leads to 


$$
\begin{aligned}
\nabla_{t}^{2} E_{z}+\frac{\beta k_{t}}{k_{t}^{2}-\beta^{2}} \frac{\mu_{g}}{\mu_{t}} \nabla_{t}^{2}\left(j \eta_{t} H_{z}\right) & =\left(\beta^{2}-k_{t}^{2}\right) E_{z} \\
\nabla_{t}^{2}\left(j \eta_{t} H_{z}\right)+\frac{\beta k_{t}}{k_{t}^{2}-\beta^{2}} \frac{\mu_{g}}{\mu_{t}} \nabla_{t}^{2} E_{z} & =j \eta_{t} H_{z} \frac{\mu_{z}}{\mu_{t}}\left(\beta^{2}-k_{t}^{2}\right)
\end{aligned}
$$

where $\eta_{t}=\sqrt{\mu_{t} / \epsilon}$ and $k_{t}=\omega \sqrt{\mu_{t} \epsilon}$. The eigenfield solution for (3) and (4) is a field, for which it holds $E_{z}=\alpha j \eta_{t} H_{z} \quad(\alpha=$ constant $)$. Thus, longitudinal field functions $E_{z}$ and $H_{z}$ differ only by a scalar factor. The special case $\mu_{t} \approx \mu_{z}=\mu$, which is achieved at higher frequency range $\omega>>\omega_{o}$, leads to $\alpha= \pm 1$, i.e., (3) and (4) can be written as

$$
\nabla_{t}^{2}\left(E_{z \pm}\right)+\left[\frac{k^{2}-\beta_{ \pm}^{2}}{1 \mp \frac{\beta_{ \pm} k}{k^{2}-\beta_{ \pm}^{2}} \frac{\mu_{g}}{\mu}}\right] E_{z \pm}=0 \quad \text { or briefly } \quad\left(\nabla_{t}^{2}+k_{c \pm}^{2}\right) E_{z \pm}=0
$$

The solutions $E_{z \pm}=\frac{1}{2}\left(E_{z} \mp j \eta H_{z}\right)$ are so-called wave-fields, often used with structures involving chiral material and elliptically polarised eigenfields [6].Corresponding transverse fields are elliptically polarised and the sign $( \pm)$ indicates the handedness: + and - correspond to right- and left-handed polarisations, respectively.

The solutions of the Helmholtz equation (5) in cylindrical coordinates are Bessel functions of the first kind

$$
E_{z \pm}=A_{n \pm} J_{n}\left(k_{c \pm} \rho\right) e^{j n \varphi}
$$

The coefficients $k_{c \pm}$ are determined by the hard-surface boundary condition at $\rho=a$ : [2]

$$
\mathbf{u}_{z} \cdot \mathbf{E}=0, \quad \mathbf{u}_{z} \cdot \mathbf{H}=\left.0 \Rightarrow E_{z \pm}\right|_{\rho=a}=0 .
$$

Using this condition with equation (6) leads to the cut-off number solution $k_{c \pm}=k_{c}=\frac{p_{n s}}{a}$, where $p_{n s}$ are the zeros of the Bessel functions. From the dispersion relation

$$
k_{c}^{2}=\frac{k^{2}-\beta_{ \pm}^{2}}{1 \mp \frac{\beta_{ \pm} k}{k^{2}-\beta_{ \pm}^{2}} \frac{\mu_{g}}{\mu}}
$$

propagation factors can be solved, still assuming $\frac{\mu_{g}}{\mu}$ small:

$$
\beta_{ \pm}=\beta_{m} \pm \beta_{d}=\frac{\beta_{0}}{1-\left(\frac{k}{\beta_{0}} \frac{\mu_{g}}{2 \mu}\right)^{2}} \pm \beta_{0} \frac{\frac{k}{\beta_{0}} \frac{\mu_{g}}{2 \mu}}{1-\left(\frac{k}{\beta_{0}} \frac{\mu_{g}}{2 \mu}\right)^{2}} .
$$

where $\beta_{0}=\sqrt{k^{2}-k_{c}^{2}}$.

\section{MODE TRANSFORMING EFFECT}

The polarisation properties of fields are considered in a circular hard-surface waveguide filled with slightly gyrotropic medium. Assuming $\frac{k}{\beta_{0}} \frac{\mu_{g}}{2 \mu}<<1$, the propagation factors can be further approximated from (9) as

$$
\beta_{ \pm}=\beta_{0} \pm \frac{k \mu_{g}}{2 \mu}
$$

Taking all the spatial dimensions into account, the + and - waves are

$$
E_{z \pm}(\rho, \varphi, z)=A_{n \pm} J_{n}\left(k_{c} \rho\right) e^{j n \varphi} e^{-j \beta_{ \pm} z}=\frac{1}{2}\left[E_{z}(\rho, \varphi, z) \mp j \eta H_{z}(\rho, \varphi, z)\right]
$$


and the total longitudinal electric and magnetic fields, due to + and - waves, are

$$
\begin{aligned}
E_{z} & =E_{z+}+E_{z-}=J_{n}\left(k_{c} \rho\right) e^{j n \varphi}\left[\left(A_{n+}+A_{n-}\right) \cos \left(\frac{k \mu_{g}}{2 \mu} z\right)-j\left(A_{n+}-A_{n-}\right) \sin \left(\frac{k \mu_{g}}{2 \mu} z\right)\right] e^{-j \beta_{0} z} \\
H_{z} & =\frac{\left(E_{z+}-E_{z-}\right)}{-j \eta}=\frac{j}{\eta} J_{n}\left(k_{c} \rho\right) e^{j n \varphi}\left[\left(A_{n+}-A_{n-}\right) \cos \left(\frac{k \mu_{g}}{2 \mu} z\right)-j\left(A_{n+}+A_{n-}\right) \sin \left(\frac{k \mu_{g}}{2 \mu} z\right)\right] e^{-j \beta_{0} z}
\end{aligned}
$$

Let us assume that a TM-mode incident field is coming from the non-gyrotropic HS waveguide section $(z<0)$. Thus, at the medium-interface $z=0$ (see Figure 1), magnetic field $H_{z}=0$. From (13) it follows that $A_{n+}=A_{n-}$, which leads to axial fields along the gyrotropic waveguide section:

$$
\begin{aligned}
& E_{z}(\rho, \varphi, z)=2 A_{n+} J_{n}\left(k_{c} \rho\right) e^{j n \varphi} e^{-j \beta_{0} z} \cos \left(\frac{k \mu_{g}}{2 \mu} z\right), \\
& H_{z}(\rho, \varphi, z)=\frac{2 A_{n+}}{\eta} J_{n}\left(k_{c} \rho\right) e^{j n \varphi} e^{-j \beta_{0} z} \sin \left(\frac{k \mu_{g}}{2 \mu} z\right) .
\end{aligned}
$$

It is seen that just after $z=0$, along the ferrite filled waveguide section, $E_{z}$ starts to decrease and $H_{z}$ starts to increase. At distance $z=d=\frac{\pi \mu}{\mu_{g} k}, E_{z}=0$ and $H_{z} \neq 0$. Thus, TM mode has changed to TE mode, which propagates after the gyrotropic section without change. The field configuration after the transformation section satisfies the boundary condition for magnetic conductor. The transformation between TM and TE modes is a dual transformation. The hard-surface waveguide can support fields which satisfy either perfect electric conductor or perfect magnetic conductor boundary conditions. In this example it was assumed that the different waveguide sections are well matched i.e. containing properly chosen media.

Assume a ferrite material with $\epsilon_{r}=8, B_{0}=0.03 \mathrm{~T}$ and relatively low saturation magnetization $\mu_{0} M_{s}=0.1 \mathrm{~T}$, i.e., $\omega_{m}=2 \pi \cdot 2.8 G H z$ (typical value is $\mu_{0} M_{s}=0.2 \mathrm{~T}$, i.e., $\omega_{m}=2 \pi \cdot 5.6 \mathrm{GHz}[5]$ ). The special case, $\mu_{t} \approx \mu_{z}=\mu_{0}$ and $\mu_{g}$ small, is obtained e.g. at $f=35$ GHz. Using formulas (2) one obtains $\mu_{t} / \mu_{z}=0.998 \approx 1$ and $\mu_{g} / \mu_{z}=0.080$. Assuming mode with $p_{n s}=p_{01}=2.405$ and core radius $a=0.0017 \mathrm{~m}$, propagation factors are approximately $\beta_{ \pm}=\beta_{0} \pm \frac{k \mu_{g}}{2 \mu} \approx(1.51 \pm 0.08) 10^{3} 1 / \mathrm{m}$. The length of the gyrotropic section, to change from TM to TE, is now $d \approx 0.019 \mathrm{~m}$.

\section{IMPEDANCE TRANSFORMER}

The wave impedance transforming effect can be studied by considering the transverse fields. The exact transverse fields in a gyrotropic waveguide are

$$
\begin{aligned}
\mathbf{h} & =\frac{1}{A^{2}+B^{2}}\left[j \beta\left(A \nabla_{t} H_{z}-B \mathbf{u}_{z} \times \nabla_{t} H_{z}\right)+j \omega \epsilon\left(A \mathbf{u}_{z} \times \nabla_{t} E_{z}+B \nabla_{t} E_{z}\right)\right] \\
\mathbf{e} & =\frac{1}{j \omega \epsilon}\left[-j \beta \mathbf{u}_{z} \times \mathbf{h}-\mathbf{u}_{z} \times \nabla_{t} H_{z}\right], \quad A=\beta^{2}-k^{2}, \quad B=j k^{2} \frac{\mu_{g}}{\mu}
\end{aligned}
$$

If $\frac{\mu_{g}}{\mu}$ is very small and $\beta$ is not near $k, \beta<k$, the gyrotropic factor $B<<A$, i.e., $B \approx 0$. If also $\frac{k}{\beta_{0}} \frac{\mu_{g}}{2 \mu}$ is small enough, the approximation $\beta=\beta_{ \pm} \approx \beta_{0}$ can be done with the factors affecting field amplitudes. These approximations lead to expressions

$$
\mathbf{e}=-j \frac{\beta_{0}}{k_{c}^{2}} \nabla_{t} E_{z}+j \frac{k \eta}{k_{c}^{2}} \mathbf{u}_{z} \times \nabla_{t} H_{z}, \quad \mathbf{h}=-j \frac{\beta_{0}}{k_{c}^{2}} \nabla_{t} H_{z}-j \frac{k}{k_{c}^{2} \eta} \mathbf{u}_{z} \times \nabla_{t} E_{z}
$$

The wave impedance $\overline{\bar{Z}}$ is the relation between the transverse fields as

$$
\mathbf{e}=-\overline{\bar{Z}} \cdot\left(\mathbf{u}_{z} \times \mathbf{h}\right)
$$

Following the example in the previous section, at $z=0$, there exists only axial electric field component $E_{z}$ and the wave impedance dyadic is $\overline{\bar{Z}}=\eta \frac{\beta_{0}}{k} \overline{\bar{I}}_{t}$. This is a $T M$ impedance. After the distance $d=\frac{\pi \mu}{\mu_{g} k}$, there exists only axial magnetic field, and the corresponding impedance dyadic is $\overline{\bar{Z}}=\eta \frac{k}{\beta_{0}} \overline{\bar{I}}_{t}$ which is 
a $T E$ impedance. At other points inside the waveguide section, $0<z<d$, there is a hybrid mode, a combination of $T E$ and $T M$ fields. The impedance dyadic is

$$
\overline{\bar{Z}}(z)=\eta \frac{\beta_{0} k \overline{\bar{I}}_{t}-\frac{k_{c}^{2}}{2} \sin \left(\frac{k \mu_{g}}{\mu} z\right) \mathbf{u}_{z} \times \overline{\bar{I}}}{k^{2} \cos ^{2}\left(\frac{k \mu_{g}}{2 \mu} z\right)+\beta_{0}^{2} \sin ^{2}\left(\frac{k \mu_{g}}{2 \mu} z\right)}
$$

In principle, with a proper length of a ferrite filled HS waveguide a hybrid mode field can be changed to $T E$ or $T M$ field. Also the impedance is transformed correspondingly. This kind of waveguide element can be used for matching between different kind of waveguides.

\section{CONCLUSION}

In a non-gyrotropic HS waveguide TE and TM modes are propagating. Using a gyrotopic HS waveguide section of a proper length, TM-mode can be transformed to TE, and vice versa. Also, the impedances are changed and the gyrotropic HS waveguide works as an impedance transformer. One application is a matching element between a circular metal-wall waveguide and an open dielectric waveguide/antenna. In this study circular waveguide was considered. Similar transformer can be implemented by using rectangular waveguide as well.
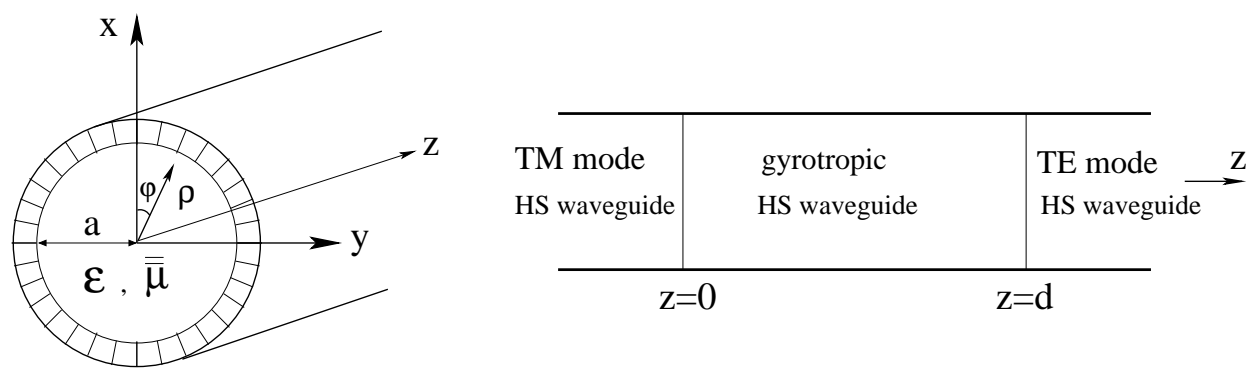

Figure 1. HS waveguide filled with gyrotropic material and TM/TE-transformer.

\section{References}

[1] P.J.B. Clarricoats, A.D. Olver, Corrugated Horns for Microwave Antennas, Stevenage: Peregrinus, 1984.

[2] P-S. Kildal, "Artificially Soft and Hard Surfaces in Electromagnetics," IEEE Trans. Antennas Propagat., Vol. 38, No. 10, pp. 1537-1544, October 1990.

[3] A.A. Kishk, M. Morgan, "Analysis of Circular Waveguides with Soft and Hard Surfaces Realized by Strip-loaded Walls Using Asymptotic Boundary Conditions", Microwave and Optical Technology Letters, Vol. 29, No. 6, pp. 433-436, June 2001.

[4] A.J. Viitanen, "Chiral Hard-Surface Waveguide Mode Transformer," IEEE Trans. Microwave Theory and Techniques, vol. 48, No. 6, pp. 1077-1079, June 2000.

[5] R.E. Collin, Foundations for Microwave Engineering, New York: McGraw-Hill, 1966.

[6] I.V. Lindell, A.H. Sihvola, S.A. Tretyakov, A.J. Viitanen, Electromagnetic Waves in Chiral and Bi-Isotropic Media, Norwood, NY: Artech House, 1994. 